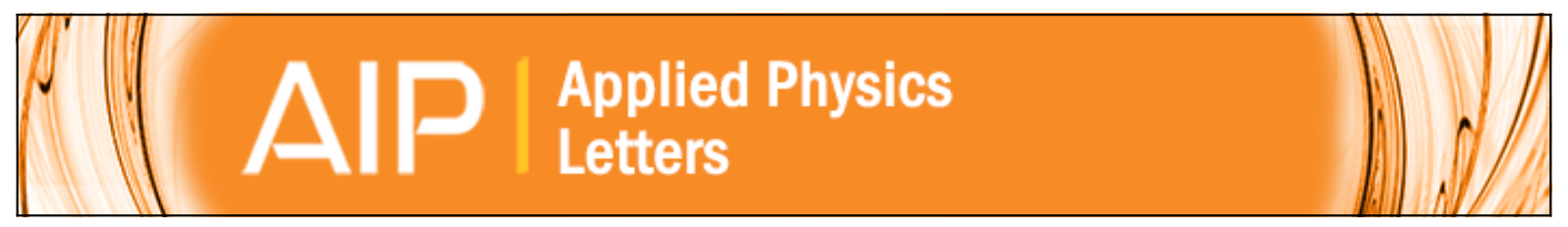

\title{
Atomic resolution on Si (111)-(7×7) by noncontact atomic force microscopy with a force sensor based on a quartz tuning fork
}

Franz J. Giessibl

Citation: Applied Physics Letters 76, 1470 (2000); doi: 10.1063/1.126067

View online: http://dx.doi.org/10.1063/1.126067

View Table of Contents: http://scitation.aip.org/content/aip/journal/apl/76/11?ver=pdfcov

Published by the AIP Publishing

\section{Articles you may be interested in}

Resonance frequency-retuned quartz tuning fork as a force sensor for noncontact atomic force microscopy Appl. Phys. Lett. 105, 043107 (2014); 10.1063/1.4891882

Atomic resolution on $\mathrm{MgO}(001)$ by atomic force microscopy using a double quartz tuning fork sensor at lowtemperature and ultrahigh vacuum

Appl. Phys. Lett. 87, 083104 (2005); 10.1063/1.2012523

Double quartz tuning fork sensor for low temperature atomic force and scanning tunneling microscopy

Rev. Sci. Instrum. 75, 2446 (2004); 10.1063/1.1765753

Atomic force microscopy of nickel dot arrays with tuning fork and nanotube probe

J. Vac. Sci. Technol. B 21, 323 (2003); 10.1116/1.1539066

Noncontact scanning force microscopy based on a modified tuning fork sensor

Rev. Sci. Instrum. 71, 3104 (2000); 10.1063/1.1304881

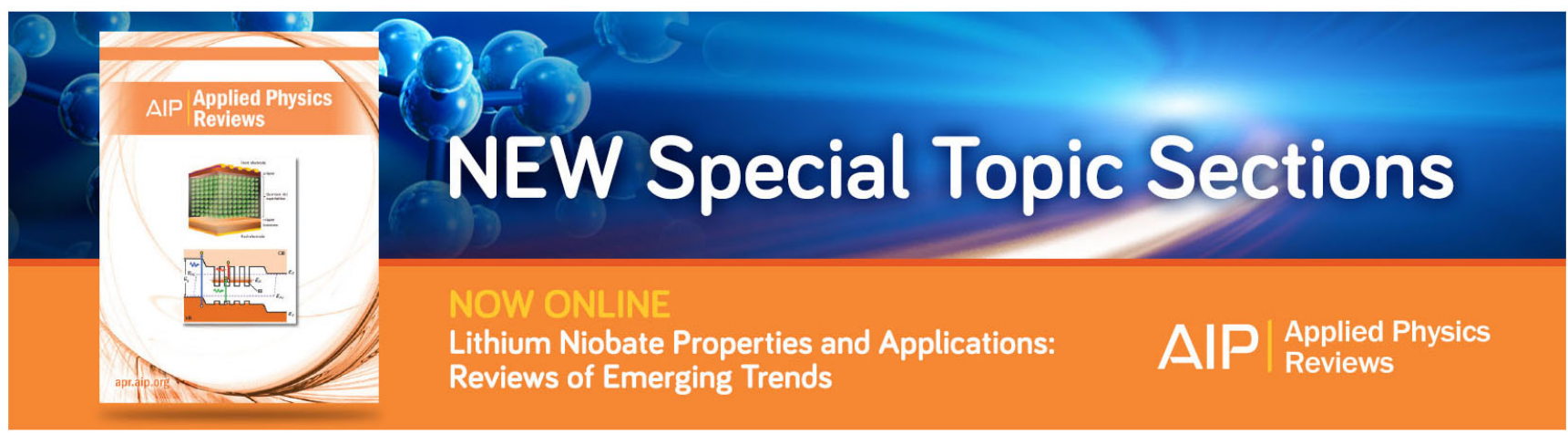




\title{
Atomic resolution on $\mathrm{Si}(111)-(7 \times 7)$ by noncontact atomic force microscopy with a force sensor based on a quartz tuning fork
}

\author{
Franz J. Giessibl ${ }^{\text {a) }}$ \\ Universität Augsburg, Institute of Physics, EKM, Experimentalphysik VI, D-86135 Augsburg, Germany
}

(Received 2 November 1999; accepted for publication 18 January 2000)

\begin{abstract}
Atomic resolution by noncontact atomic force microscopy with a self-sensing piezoelectric force sensor is presented. The sensor has a stiffness of $1800 \mathrm{~N} / \mathrm{m}$ and is operated with sub-nanometer amplitudes, allowing atomic resolution with relatively bluntly etched tungsten tips. Sensitivity and noise are discussed. (C) 2000 American Institute of Physics. [S0003-6951(00)01011-1]
\end{abstract}

The force sensing cantilever (CL) is a central component of an atomic force microscope (AFM). ${ }^{1}$ The CL can be characterized by three parameters: stiffness $k$, eigenfrequency $f_{0}$, and quality factor $Q .{ }^{2}$ In static AFM, it has been estimated that $k$ should range from 0.01 to $1 \mathrm{~N} / \mathrm{m}^{3}{ }^{3}$ When atomic resolution in vacuum is desired, ${ }^{4}$ the preferred method is frequency modulation (FM) AFM. ${ }^{5}$ In this method, a CL with $k \approx 20 \mathrm{~N} / \mathrm{m}, f_{0} \approx 100 \mathrm{kHz}, Q \approx 10^{4}$ oscillates with an amplitude $A \approx 10 \mathrm{~nm}$ (see Table I in Ref. 6). The initial values for $k$ and $A$ have been found empirically and a theoretical study has shown that maximum signal-to-noise ratio should be obtained with $A \approx 0.3-1 \mathrm{~nm} .{ }^{6}$ For achieving stable oscillation with such small amplitudes, the spring constant $k$ of the CL must be of the order of a few hundred newtons per meter. These findings have motivated the attempt to achieve atomic resolution with quartz tuning forks with a stiffness of a few kilonewtons per meter. Quartz tuning forks are produced mainly for watches with an annual production volume $>2$ $\times 10^{9}{ }^{7}$ Economic and practical considerations within the watch industry have helped to shrink their size such that they are now appropriate as AFM sensors. Like piezoresistive sensors, ${ }^{8}$ quartz tuning forks have the advantage of selfsensing, i.e., the use of optics is not necessary and operation in ultrahigh vacuum and low temperatures are easily implemented. Tuning forks have been used in scanning probe microscopes before. $^{9-17}$ However, atomic resolution has not been reported so far.

Our microscope is a modified commercial scanning tunneling microscope (STM). ${ }^{18}$ The vacuum chamber is pumped with an ion and titanium sublimation pump. The sample is a $11 \times 14 \mathrm{~mm}^{2}$ piece of a silicon (111) wafer. It is prepared by heating it to $1300{ }^{\circ} \mathrm{C}$ for $30 \mathrm{~s}$ with an electron beam heater while maintaining a pressure $p<5 \times 10^{-9}$ mbar. The unique approach of this tuning fork application is that here one of the prongs is held fixed ["qPlus" configuration, ${ }^{15}$ Fig. 1(a)]. Three improvements over the original approach ${ }^{15}$ have resulted in a dramatic increase in resolution:

(a) a quartz stabilized, digital FM demodulator ${ }^{19}$ is utilized;

(b) the deflection of the sensor is measured by the current which is required to keep the two electrodes at constant potential [see Fig. 1(b)]—previously, the voltage across the

${ }^{a)}$ Electronic mail: Franz.Giessibl@Physik.Uni-Augsburg electrodes was measured with an instrumentation amplifier; and

(c) a custom made substrate for the tuning forks is used now which facilitates manufacturing and handling of the sensors [see Fig. 1(a)].

We use quartz tuning forks with $f_{0}^{\text {bare }}=32768 \mathrm{~Hz}$, the length of one beam is $L=2.4 \mathrm{~mm}$, thickness $t=214 \mu \mathrm{m}$, and width $w=130 \mu \mathrm{m}$. Thus, the theoretical spring constant is $k \approx 1800 \mathrm{~N} / \mathrm{m}$. ${ }^{15}$ One of the prongs is glued to a substrate and an etched tungsten tip is attached to the other prong with silver expoxy [Fig. 1(a)]. The mass of the tungsten tip causes $f_{0}$ to drop to typical values between 15 and $30 \mathrm{kHz}$. The electrode which is connected to the tip can be kept at a constant potential $V_{t}$, thus the sensor can be used for STM and simultaneous STM/AFM by collecting the tunneling current at the sample.

The operation of the sensor is based on the piezoelectric effect: bending the end of the CL by $z^{\prime}$ causes strain in the CL [see Fig. 1(b)]. The strain causes surface charges which are collected by the metal electrodes. For determining the sensitivity $S$ (defined as $V_{\text {out }} / z^{\prime}$ ), three methods were used and cross checked: (a) calculation, (b) analysis of the thermal
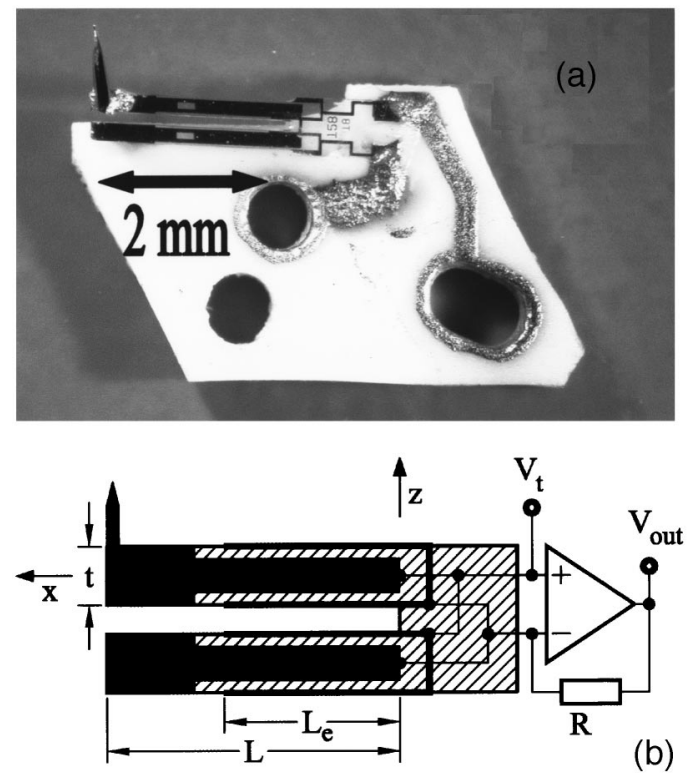

(b)

FIG. 1. (a) Photograph of the force sensor: one of the prongs and the base part of a quartz tuning fork are attached to a substrate, a W tip is mounted to the other prong and (b) Schematic of electrodes and preamplifier. 


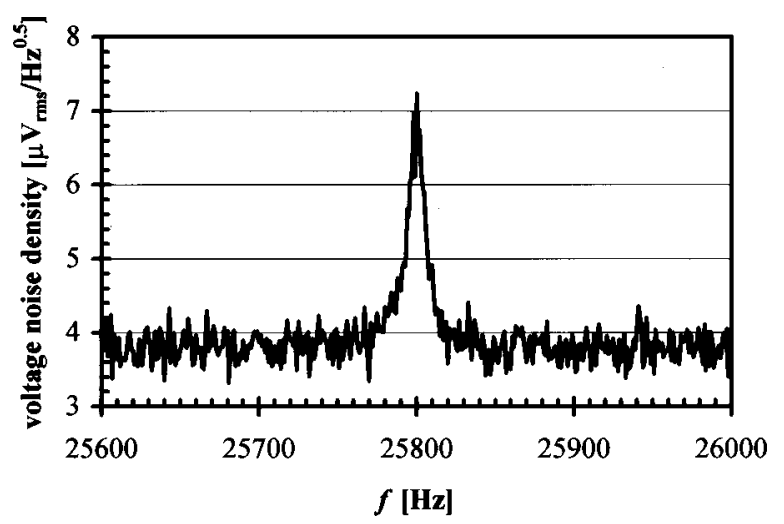

FIG. 2. Noise spectrum of the sensor. The peak corresponds to the thermal oscillation with an amplitude of $A=1.5 \mathrm{pm}_{\mathrm{rms}}$.

noise spectrum, and (c) measuring a zero-size image with varying amplitude.

(a) When a force $F=k z^{\prime}$ is acting on the upper prong [Fig. 1(b)], the strain $\epsilon$ at the upper side is given by

$$
\epsilon(x, z=t / 2)=\frac{t}{2} F(x-L) \frac{1}{E J},
$$

where $E$ is Young's modulus and $J$ is the moment of inertia $J=w t^{3} / 12$. This strain causes a stress $\sigma_{\text {mech }}=\epsilon E$ which leads to a surface charge density $\sigma_{\text {charge }}=\sigma_{\text {mech }} d_{21}$ where $d_{21}$ is the piezoelectric coupling constant $\left(d_{21}=2.31 \times 10^{-12} \mathrm{C} / \mathrm{N}\right.$ for quartz). ${ }^{20}$ The lower side of the bent prong also has a charge density $\sigma_{\text {charge }}$ (both $\epsilon$ and the $z$ component of the surface normal vector have opposite signs) and contributes an equal amount to the total charge $q$. Integrating $\sigma_{\text {charge }}$ from $x=0$ to $x=L_{e} \quad(=$ length of the electrodes $)$ and $y=-w / 2$ to $w / 2$ yields

$$
q / z^{\prime}=12 d_{21} k L_{e}\left(L_{e} / 2-L\right) / t^{2} .
$$

With $z^{\prime}=A \cos (2 \pi f \tau)$ and $L_{e} \approx 1.6 \mathrm{~mm}$ the sensitivity is $S$ $\approx 2 \pi f \times R \times 2.8 \mu \mathrm{C} / \mathrm{m}$ and with $f=25800 \mathrm{~Hz}$ and $R$ $=100 \mathrm{M} \Omega$ we find $S_{\text {theory }} \approx 45 \mu \mathrm{V} / \mathrm{pm}$.

(b) The thermal oscillation of the CL can be used to calibrate its sensitivity: according to the equipartition theorem, $k\left\langle z_{\text {th }}^{\prime 2}\right\rangle=k_{B} T$, thus the thermal oscillation amplitude $A_{\text {th_rms }}=\sqrt{k_{B} T / k}$ where $k_{B}$ is the Boltzmann constant and $T$ is the temperature in kelvin. For $T=300 \mathrm{~K}, A_{\text {th_rms }}$ $=1.5 \mathrm{pm}$. Figure 2 shows a thermal noise spectrum of $V_{\text {out }}$ at room temperature with $f=25800 \mathrm{~Hz}$ and $R=100 \mathrm{M} \Omega$. The thermal noise amplitude corresponds to $V=33 \mu \mathrm{V}_{\text {rms }}$, resulting in an experimental sensitivity $S_{\exp 1} \approx 22 \mu \mathrm{V} / \mathrm{pm}$.

(c) A second experimental method to find the sensitivity is based on taking a zero-size image with slow acquisition speed. The first half of the image is acquired with amplitude $A_{1}$ and frequency shift $\Delta f_{1}$ and the second half with $A_{2}$ $=2^{2 / 3} A_{1}$ and $\Delta f_{2}=0.5 \Delta f_{1}$. Since $\Delta f \propto A^{-3 / 2}$ for a constant distance $d$ between the sample and the lower turnaround point of the CL, ${ }^{21} d$ is the same for the parameter sets $\left(\Delta f_{1}, A_{1}\right)$ and $\left(\Delta f_{2}, A_{2}\right)$ and the height of the step which occurs in the image is equivalent to the difference in amplitudes $A_{1}-A_{2}$. This method yields an experimental sensitivity $S_{\exp 2}=20.9 \mu \mathrm{V} / \mathrm{pm}$.

The experimental sensitivities derived by methods (b) and (c) show excellent agreement. The theoretical sensitivity

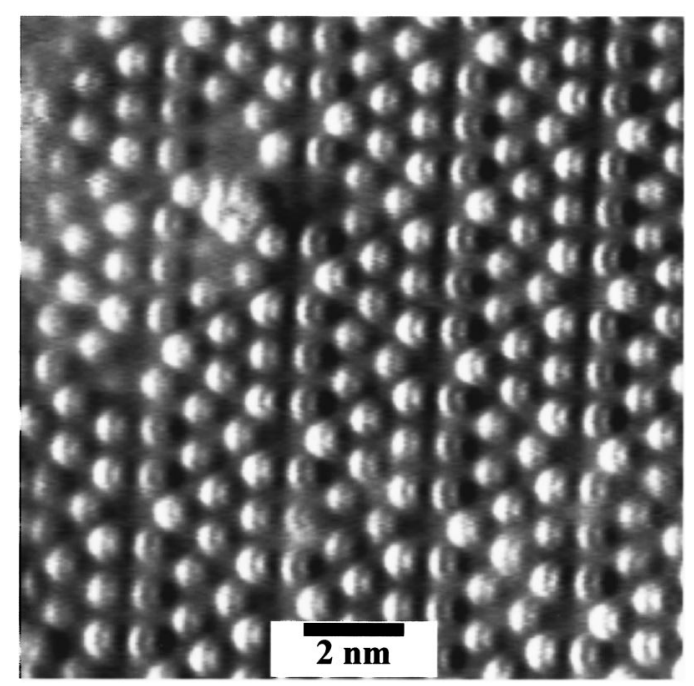

FIG. 3. Noncontact topographic AFM image (raw data) of Si(111)$(7 \times 7)$. Some adatoms appear to be missing in the upper left section of the image.

also agrees well with the experimental values. Possibly, the limited gain-bandwidth product of the current/voltage $(I / V)$ converter, stray capacitances and edge effects in the tuning forks (the width of the electrodes is finite) cause the deviation between experiment and theory. The spectral noise density of the sensor is $170 \mathrm{fm} / \sqrt{\mathrm{Hz}}$ (estimated with $S_{\exp 1}$ and the noise floor in Fig. 2). The thermal limit is given by the Johnson noise of the resistor $R(1.3 \mu \mathrm{V} / \sqrt{\mathrm{Hz}} \triangleq 60 \mathrm{fm} / \sqrt{\mathrm{Hz}})$ at room temperature. At $T=4 \mathrm{~K}$, the lower limit for the deflection noise density is $10 \mathrm{fm} / \sqrt{\mathrm{Hz}}$ which is far less than the noise density in optical detection schemes. ${ }^{2}$

The sensor was used to obtain a topographic image $(\Delta f$ constant) of $\mathrm{Si}(111)-(7 \times 7)$ with a size of $10 \times 10 \mathrm{~nm}^{2}$ (Fig. 3). The parameters used were $\Delta f=-160 \mathrm{~Hz}, f_{0}$ $=16860 \mathrm{~Hz}, A=0.8 \mathrm{~nm}$, sample bias $+1.6 \mathrm{~V}$ and acquisition speed 4 lines/s. This image is also an experimental verification of the validity of the "normalized" frequency shift $\gamma=k A^{3 / 2} \Delta f / f_{0}^{21}$ as the relevant parameter in noncontact AFM. Lüthi et $a .^{22}$ have performed traditional (i.e., large $A$, small $k$, sharp tips) noncontact AFM measurements on $\mathrm{Si}(111)-(7 \times 7)$ with a sample bias $+1.8 \mathrm{~V}, \Delta f$ $=-450 \mathrm{~Hz}, f_{0}=153 \mathrm{kHz}, A=19 \mathrm{~nm}$, and $k=23.5 \mathrm{~N} / \mathrm{m}$, yielding $\gamma=-181 \mathrm{fN} \sqrt{\mathrm{m}}$. Here, $\gamma$ is twice as large $(-386 \mathrm{fN} \sqrt{\mathrm{m}})$ which is due to enhanced electrostatic and van-der-Waals interaction of etched $\mathrm{W}$ tips versus micromachined $\mathrm{Si}$ tips (the tip radius of $\mathrm{W}$ tips is typically much greater than the radius of commercial $\mathrm{Si} C L$ tips). Even though $k$ and $A$ differ by orders of magnitude, $\gamma$ is comparable for these two experiments.

In summary, atomic resolution with a force sensor based on a quartz tuning fork was demonstrated. The sensitivity $S$ has been calculated and two experimental methods for measuring $S$ have been introduced. The advantages of this sensor over conventional CLs are: (i) large $k$ allows oscillation with small amplitudes which enhances sensitivity to short-range forces $^{23}$ yielding atomic resolution with conventional tungsten tips (demonstrated by Erlandsson et al. ${ }^{24}$ ), small $A$ 's allow simultaneous STM/AFM; (ii) piezoelectric detection scheme is easy to implement in adverse environments (vacuum, low temperature) ${ }^{10}$ and has extremely low power 
dissipation (picowatts instead of milliwatts); (iii) due to the small size and weight of the sensor standard STMs can be easily extended to combined STMs/AFMs; and (iv) high $Q$ value in air allows FM-AFM in ambient conditions. ${ }^{15}$

The author wishes to thank Nanosurf AG for supplying the digital FM demodulator and tuning forks and ThermoMicroscopes for components for our microscope head. The author also thanks Klaus Wiedenmann for help with the design of our vacuum system and microscope head, Stefan Hembacher and Hartmut Bielefeldt for discussions, Douglas P. E. Smith for sharing design ideas for the $I / V$ converter, and Jochen Mannhart for support and editorial advice. This work is funded by BMBF (Project No. 13N6918/1).

${ }^{1}$ G. Binnig, C. F. Quate, and Ch. Gerber, Phys. Rev. Lett. 56, 930 (1986).

${ }^{2}$ D. Sarid, Scanning Force Microscopy (Oxford University Press, New York, 1994).

${ }^{3}$ D. Rugar and P. Hansma, Phys. Today 43, 23 (1990).

${ }^{4}$ F. J. Giessibl, Science 267, 68 (1995); S. Kitamura, M. Iwatsuki, Jpn. J. Appl. Phys., Part 2 34, L145 (1995).

${ }^{5}$ T. R. Albrecht, P. Grütter, D. Horne, and D. Rugar, J. Appl. Phys. 69, 668 (1991).

${ }^{6}$ F. J. Giessibl, H. Bielefeldt, S. Hembacher, and J. Mannhart, Appl. Surf. Sci. 140, 352 (1999).

${ }^{7}$ E. Momosaki, Proc. 1997 IEEE Interntl. Freq. Contr. Symp., 552 (IEEE, New York, 1997), p. 552.
${ }^{8}$ M. Tortonese, R. C. Barrett, and C. F. Quate, Appl. Phys. Lett. 62, 834 (1993).

${ }^{9}$ P. Guethner, U. Fischer, and K. Dransfeld, Appl. Phys. B: Photophys. Laser Chem. 48, 89 (1989).

${ }^{10}$ K. Karrai and R. D. Grober, Appl. Phys. Lett. 66, 1842 (1995).

${ }^{11}$ A. G. T. Ruiter, J. A. Veerman, K. O. van der Werf, and N. F. van Hulst, Appl. Phys. Lett. 71, 28 (1997).

${ }^{12}$ H. Edwards, L. Taylor, W. Duncan, and A. Melmed, J. Appl. Phys. 82, 980 (1997).

${ }^{13}$ M. Todorovic and S. Schulz, J. Appl. Phys. 83, 6229 (1998).

${ }^{14}$ D. P. Tsai and Y. Y. Lu, Appl. Phys. Lett. 73, 2724 (1998).

${ }^{15}$ F. J. Giessibl, Appl. Phys. Lett. 73, 3956 (1998).

${ }^{16}$ J. Rychen, T. Ihn, P. Studerus, A. Herrmann, and K. Ensslin, Rev. Sci. Instrum. 70, 2765 (1999).

${ }^{17}$ W. H. J. Rensen, N. F. van der Hulst, A. G. T. Ruiter, and P. E. West, Appl. Phys. Lett. 75, 1640 (1999).

${ }^{18}$ SU-2 microscope head with AutoProbe electronics, Thermo Microscopes, Sunnyvale, California.

${ }^{19}$ Nanosurf AG, Liestal, Switzerland.

${ }^{20}$ C. J. Chen, Introduction to Scanning Tunneling Microscopy (Oxford University Press, New York, 1993).

${ }^{21}$ F. J. Giessibl, Phys. Rev. B 56, 16010 (1997).

${ }^{22}$ R. Lüthi, E. Meyer, M. Bammerlin, A. Baratoff, T. Lehmann, L. Howald, Ch. Gerber, and H. Guntherodt, Z. Phys. B: Condens. Matter 100, 165 (1996).

${ }^{23}$ F. J. Giessibl and H. Bielefeldt, Phys. Rev. B (in press).

${ }^{24}$ R. Erlandsson, L. Olsson, and P. Martensson, Phys. Rev. B 54, R8309 (1996). 